\title{
Hapalindoles from the Cyanobacterium Hapalosiphon sp. Inhibit T Cell Proliferation
}

Authors

Tomasz Chilczuk $^{1 *}$, Carmen Steinborn ${ }^{2 *}$, Steffen Breinlinger ${ }^{1 *}$, Amy Marisa Zimmermann-Klemd ${ }^{2}$, Roman Huber ${ }^{2}$, Heike Enke ${ }^{3}$, Dan Enke ${ }^{3}$, Timo Horst Johannes Niedermeyer ${ }^{1 \S}$, Carsten Gründemann ${ }^{\S}$

\section{Affiliations}

1 Department of Pharmaceutical Biology/Pharmacognosy, Institute of Pharmacy, University of Halle-Wittenberg, Halle, Germany

2 Center for Complementary Medicine, Institute for Infection Prevention and Hospital Epidemiology, Faculty of Medicine, University of Freiburg, Freiburg, Germany

3 Cyano Biotech GmbH, Berlin, Germany

\section{Key words}

cyanobacteria, Hapalosiphon, hapalindoles, immune modulation, lymphocytes

received June 6, 2019

revised November 5, 2019

accepted November 6, 2019

Bibliography

DOI https://doi.org/10.1055/a-1045-5178

Published online November 27, 2019 | Planta Med 2020; 86: 96-103 @ Georg Thieme Verlag KG Stuttgart · New York | ISSN 0032-0943

\section{Correspondence}

PD Dr. Carsten Gründemann

Center for Complementary Medicine, Institute for Infection Prevention and Hospital Epidemiology, Faculty of Medicine Breisacherstrasse 115B, 79106 Freiburg, Germany Phone: + 49(0) 76127083170 , Fax: + 49(0) 76127083230 carsten.gruendemann@uniklinik-freiburg.de

\section{Correspondence}

Prof. Dr. Timo H.J. Niedermeyer

Department of Pharmaceutical Biology/Pharmacognosy, Institute of Pharmacy

Hoher Weg 8, 06120 Halle (Saale), Germany

Phone: + 49(0) 3455525765 , Fax: + 49(0) 3455527407

timo.niedermeyer@pharmazie.uni-halle.de
Supporting information available online at

http://www.thieme-connect.de/products

\section{ABSTRACT}

Novel immunomodulating agents are currently sought after for the treatment of autoimmune diseases and cancers. In this context, a screening campaign of a collection of 575 cyanobacteria extracts for immunomodulatory effects has been conducted. The screening resulted in several active extracts. Here we report the results of subsequent studies on an extract from the cyanobacterium Hapalosiphon sp. CBT1235. We identified 5 hapalindoles as the compounds responsible for the observed immunomodulatory effect. These indole alkaloids are produced by several strains of the cyanobacterial family Hapalosiphonaceae. They are known for their anti-infective, cytotoxic, and other bioactivities. Modulation of the activity of human immune cells has not yet been described. The immunomodulatory activity of the hapalindoles was characterized in vitro using flow cytometry-based measurements of T cell proliferation after carboxyfluorescein diacetate succinimidyl ester staining, and apoptosis and necrosis induction after annexin $\mathrm{V} /$ propidium iodide staining. The most potent compound, hapalindole $\mathrm{A}$, reduced $\mathrm{T}$ cell proliferation with an $\mathrm{IC}_{50}$ of $1.56 \mu \mathrm{M}$, while relevant levels of apoptosis were measurable only at 10 -fold higher concentrations. Hapalindole A-formamide and hapalindole J-formamide, isolated for the first time from a natural source, had much lower activity than the nonformylated derivatives while, at the same time, being less selective for antiproliferative over apoptotic effects. 
ABBREVIATIONS

CFSE carboxyfluorescein diacetate succinimidyl ester

CPT camptothecin

CsA cyclosporine A

PBMC peripheral blood mononuclear cells

\section{Introduction}

Cyanobacteria are an intriguing source for structurally diverse and biologically active natural products. Especially the genera Microcystis, Nostoc, and Lyngbya or Moorea are chemically well-characterized [1-7]. Several strains of the family Hapalosiphonaceae produce indole alkaloids [8]. The major classes of these indole alkaloids include hapalindoles, ambiguines, fischerindoles, and welwitindolinones, of which more than 80 variants have been described in the literature [9-24]. They share several common motifs: an indole or oxindole core, a cyclohexane fused to that core, an isonitrile or isothiocyanate functional group, a chlorine substituent, or an additional ring [25]. The largest group of these alkaloids are the hapalindoles. So far 30 different hapalindoles have been reported $[9,10,15,18,21,26]$. The first hapalindoles, hapalindoles A and B, were discovered in 1984 by Moore et al. from Hapalosiphon fontinalis [9]. Since then, diverse hapalindoles have been isolated from various strains of the genera Hapalosiphon, Fischerella, and Westiellopsis $[9,17,21,26]$.

Hapalindoles have a broad spectrum of biological activities, e.g., activity against bacteria [17, 19,21,27], fungi [19,27], and algae [27]. Studies by Doan et al. suggested inhibition of RNA polymerase and consequently the disturbance of the protein biosynthesis as a possible mode of action for the antibacterial activity $[27,28]$. Furthermore, cytotoxic activity against normal mammalian and cancer cell lines has been reported for various hapalindoles [21,27]. However, no mode of action for this activity has been described yet. Finally, they have been shown to be toxic to insects $[18,29]$ and vertebrates [23]. The insecticidal activity could be explained by sodium channel modulating activity of the indole alkaloids [30]. Interestingly, inhibition of sodium channels did not lead to any cytotoxicity in neuroblastoma cell lines. Thus, compared to insects, a different mode of action must underlie the cytotoxicity on mammalian cells. Although various bioactivities can be attributed to hapalindoles, modulation of the activity of human immune cells has not yet been described. Immunomodulatory drugs are used as modifiers of the immune system to either enhance the immune response against infectious diseases, tumors, and immunodeficiency, or to suppress the immune reaction in organ transplants or to treat autoimmune responses. Screening of a cyanobacteria extract collection (575 extracts) derived from strains from all cyanobacteria orders for inhibition of T cell proliferation resulted in 35 extracts with an activity at $1 \mu \mathrm{g} /$ $\mathrm{mL}$ or lower. One of the active extracts was derived from a Hapalosiphon sp. strain. Bioassay-guided fractionation of the extract led to the isolation of 3 known hapalindoles, hapalindole $A$ (1) [9], D (2), and M (3) [10], as well as 2 formamide-bearing hapalindoles that are reported here for the first time from a natural source, namely hapalindole A-formamide (4) [31] and hapalindole J-formamide (5) [32]. Here, we report the isolation, structure elucidation, and the investigation of the immunomodulatory properties of the hapalindole derivatives isolated from Hapalosiphon sp. CBT1235.

\section{Results and Discussion}

Our initial screening of a cyanobacteria extract collection for immunomodulating activity on human immune cells resulted in 35 extracts with an activity at a concentration of $1 \mu \mathrm{g} / \mathrm{mL}$ or lower. Nine out of the 11 most potent extracts were derived from cyanobacteria of the genus Nostoc. Dereplication of the natural products in these Nostoc strain extracts showed the presence of the well-known cytotoxic compound cryptophycin-1 in most of these strains [33-36], so they were excluded from further investigation. Chromatographic evaluation of the remaining extracts by HPLC-DAD/MS showed that few of them featured prominent peaks. In the HPLC-DAD chromatogram of an Hapalosiphon sp. extract, several prominent peaks were observed; thus, this strain was selected for follow-up work. T cell proliferation was inhibited by this extract with an $\mathrm{IC}_{50}$ of $0.11 \mu \mathrm{g} / \mathrm{mL}$ ( $\vee$ Fig. 1 a). At the same time, a trend to an increased amount of apoptotic cells at an extract concentration of $10 \mu \mathrm{g} / \mathrm{mL}$ could be observed (statistically non-significant, > Fig. $\mathbf{1}$ b). No induction of necrosis has been observed (data not shown). Microfractionation of the extract and subsequent bioassays showed that indeed the major compounds observable in the chromatogram were responsible for the bioactivity and led to the significant retardation of $\mathrm{T}$ cell proliferation (Fig. 1S a-c in the Supporting Information).

Five compounds present in the active micro-fraction were isolated from the extract by semi-preparative HPLC (1-5). ${ }^{1} \mathrm{H}$ NMR spectra of the pure compounds displayed typical signals of hapalindoles. The molecular formulas were deduced from HRESIMS data. The structures of 1-5 were elucidated based on 1D and 2D NMR data. NMR and MS spectral data of compounds 1-3 matched the published data for hapalindole A (1), hapalindole D (2), and hapalindole $\mathrm{M}(3)$, respectively (structures of all isolated compounds, see - Fig. 2). NOESY experiments as well as the determination of specific rotation values confirmed the absolute configuration of compounds $1-3$ as originally described in detail by Moore et al. [10]. Hapalindole A (1) was the main compound isolated from Hapalosiphon sp. CBT1235.

Compounds 4 and 5 were hapalindole derivatives not yet described as natural products. Compound 4 was isolated as a brown oil. HRESIMS showed an $[\mathrm{M}+\mathrm{H}]^{+}$ion with $\mathrm{m} / \mathrm{z} 357.1723$, corresponding to the molecular formula $\mathrm{C}_{21} \mathrm{H}_{25} \mathrm{~N}_{2} \mathrm{OCl}$ (calcd. $357.1728, \Delta 1.54$ ppm). The ${ }^{1} \mathrm{H}$-spectrum of 4 was almost identical with the respective spectrum of 1 but with 2 additional peaks in the low-field region (8.04 ppm and $8.57 \mathrm{ppm}$ ) and a deshielded $\mathrm{H}-11$ (4.84 ppm) compared to 1 (4.37 ppm), suggesting C- 11 to be substituted with a formamide moiety ( $\bullet$ Table 1 ). Key HMBC and COSY correlations confirmed the planar structure $(\triangleright$ Fig. 3 ). Evaluation of the NOESY spectrum showed the relative configuration of 4 to be the same as hapalindole $A$ with the formamide group being attached axially to $\mathrm{C}-11$ (strong correlations of the $\mathrm{N}$-formamide proton with $\mathrm{H}-10, \mathrm{H}-13$ and $\mathrm{H}-15$ ) [9]. Comparing the specific rotation 

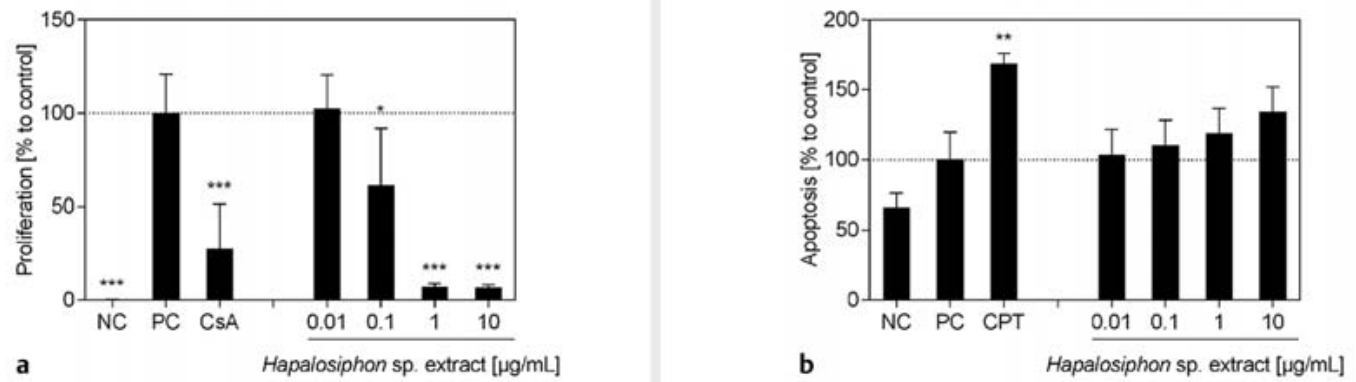

- Fig. 1 Effects of Hapalosiphon sp. CBT1235 extract on T cell proliferation and apoptosis induction. Primary human lymphocytes were cultured in the presence of medium (NC) or were stimulated with anti-human CD3 and anti-human CD28 mAb (PC; $100 \mathrm{ng} / \mathrm{mL}$ ). Activated T cells were further incubated with cyclosporine $A(C s A ; 5 \mu \mathrm{g} / \mathrm{mL})$, camptothecin (CPT; $30 \mu \mathrm{g} / \mathrm{mL})$, or different concentrations of the Hapalosiphon sp. CBT1235 extract. Cell division analysis was carried out using CFSE staining and flow cytometry. Levels of apoptosis were determined using flow-cytometric analysis of annexin V-stained cells. a Effects of Hapalosiphon sp. CBT1235 extract on lymphocyte proliferation. b Induction of apoptosis by Hapalosiphon sp. CBT1235 extract. Data of 4 (a) or 3 (b) independent experiments are presented as mean \pm SD in relation to stimulated T cells $(P C=100 \%)$. Asterisks indicate significant differences from PC controls $\left({ }^{* *} \mathrm{p}<0.01,{ }^{* * *} \mathrm{p}<0.001\right)$.

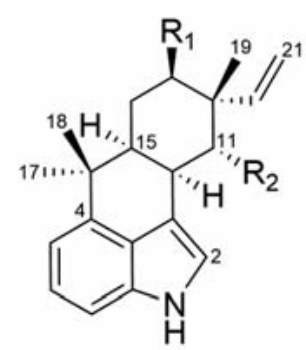

$1 \mathrm{R}_{1}=\mathrm{Cl} \mathrm{R}_{2}=\mathrm{NC}$

$3 \mathrm{R}_{1}=\mathrm{H} \mathrm{R}_{2}=\mathrm{NCS}$

$4 \mathrm{R}_{1}=\mathrm{Cl} \mathrm{R}_{2}=\mathrm{NHCHO}$

$5 \quad \mathrm{R}_{1}=\mathrm{H} \mathrm{R}_{2}=\mathrm{NHCHO}$

- Fig. 2 Chemical structures of hapalindoles 1-5 isolated from Hapalosiphon sp. CBT 1235.

values with already published data confirmed our assignment of the absolute configuration of 4 [37]. Therefore, compound 4 was identified as the new natural product hapalindole A-formamide.

The main difference with 5 compared to 1 and 4 was the absence of the chlorine. Compound 5 was also isolated as a brown oil. HRESIMS showed an $[\mathrm{M}+\mathrm{H}]^{+}$ion with $\mathrm{m} / \mathrm{z} 323.2114$, corresponding to the molecular formula $\mathrm{C}_{21} \mathrm{H}_{26} \mathrm{~N}_{2} \mathrm{O}$ (calcd. 323.2118, $\Delta 1.08 \mathrm{ppm}$ ). Evaluation of the NMR spectra confirmed the presence of a formamide group at C-11 of the hapalindole backbone, as well ( $\triangleright$ Fig. 3 and Table $\mathbf{1}$ ). The absolute configuration of $\mathbf{5}$ matches the one of hapalindole J, the nonchlorinated variant of hapalindole $A(1)$, and has been confirmed by comparing the NOESY correlations and the specific rotation values with those originally published by Moore et al. [10]. Additional NOESY correlations for the axial formamide could be observed ( $\bullet$ Fig. 4). Com- pound 5 was thus confirmed to be the new natural product hapalindole J-formamide.

Coupling constants between $\mathrm{H}-22(\mathrm{NH})$ and $\mathrm{H}-23$ were found to be $1.37 \mathrm{~Hz}$ and $1.45 \mathrm{~Hz}$ for hapalindole A-formamide (4) and $J$-formamide (5), respectively, indicating a cis conformation of the amide bond in both compounds [31].

Hapalindole formamides have been described as intermediates during the total synthesis of hapalindoles, as derivatization products with formic acid, and they can also form during storage in $d$ chloroform $[13,31,32,38]$. Here, we report for the first time the isolation of 2 hapalindole formamides as natural products from Hapalosiphon sp. CBT1235. 4 and 5 could readily be detected by HPLC-MS in a fresh Hapalosiphon sp. CBT1235 extract that has not been in contact with formic acid or other acids (Fig. 2S, Supporting Information), ruling out the possibility that the isolated formamides are processing or isolation artifacts.

All isolated hapalindoles showed significant effects in the T cell proliferation assay ( $\bullet$ Fig. 5). Hapalindole A (1) displayed the highest antiproliferative activity with an $\mathrm{IC}_{50}$ of $1.56 \mu \mathrm{M}$. Hapalindoles $D(2)$ and M (3) showed a weaker activity and suppressed proliferation with an $\mathrm{IC}_{50}$ value of $27.15 \mu \mathrm{M}$ for hapalindole D. Hapalindoles A-formamide (4) and J-formamide (5) again showed lower activity ( $\bullet$ Fig. 5). Except for hapalindole J-formamide, all hapalindoles induced T cell apoptosis at highest concentrations ( $\bullet$ Fig. $\mathbf{6}$ ).

Although relevant amounts of T cell apoptosis were detected, retardation of $\mathrm{T}$ cell proliferation was measurable at up to 10 -fold lower concentrations. The most potent compound, hapalindole A, was effective at a concentration of $3.0 \mu \mathrm{M}$ without showing cytotoxic effects. Therefore, potentially a therapeutic range for an application as an anti-inflammatory remedy is given. The isonitrile functional group seems to be crucial for the antiproliferative bioactivity of the hapalindoles, as the formamide derivatives possess a weaker activity. This is in agreement with previous findings, where a reduced antifungal and antibacterial activity of the hapalindole formamides compared to their isonitrile and isothiocyanate counterparts has been reported [31]. Our results, therefore, 
- Table $1{ }^{1} \mathrm{H}$ and ${ }^{13} \mathrm{C}$ NMR assignments for 4 and $5(600 \mathrm{MHz}$ for ${ }^{1} \mathrm{H}, 150 \mathrm{MHz}$ for ${ }^{13} \mathrm{C}$, DMSO- $\left.\mathrm{d}_{6}\right) .{ }^{13} \mathrm{C}$ chemical shifts for 5 were extracted from the $2 \mathrm{D}$ spectra.

\begin{tabular}{|c|c|c|c|c|}
\hline \multirow{2}{*}{$\begin{array}{l}\text { Posi- } \\
\text { tion }\end{array}$} & \multicolumn{2}{|l|}{4} & \multicolumn{2}{|l|}{5} \\
\hline & $\delta_{\mathrm{H}}(\mathrm{J}$ in $\mathrm{Hz})$ & $\delta_{c}$ & $\delta_{\mathrm{H}}(\mathrm{J}$ in $\mathrm{Hz})$ & $\delta_{c}$ \\
\hline 1 & $10.81, \mathrm{~s}$ & indole N & $10.69, \mathrm{~s}$ & indole $\mathrm{N}$ \\
\hline 2 & $7.24, \mathrm{t}(1.8)$ & 120.5 & $7.13, \mathrm{~m}$ & 119.7 \\
\hline 3 & & 110.6 & & 111.8 \\
\hline 4 & & 137.1 & & 138.0 \\
\hline 5 & $6.81, \mathrm{~d}(7.0)$ & 112.3 & $\begin{array}{l}6.79, \text { br d } \\
(7.02)\end{array}$ & 111.9 \\
\hline 6 & $7.02, \mathrm{t}(7.6)$ & 121.9 & $6.99, \mathrm{t}(7.4)$ & 121.5 \\
\hline 7 & 7.14, d (7.9) & 108.7 & $7.10, d(8.0)$ & 108.1 \\
\hline 8 & & 133.3 & & 133.4 \\
\hline 9 & & 124.0 & & 124.4 \\
\hline 10 & 3.29 & 36.7 & 3.30 & 36.3 \\
\hline 11 & $4.84, \mathrm{~m}$ & 54.6 & $4.59, \mathrm{~s}$ & 51.6 \\
\hline 12 & & 44.6 & & 39.1 \\
\hline $13 \mathrm{ax}$ & $\begin{array}{l}4.61, \mathrm{dd} \\
(12.4,4.0)\end{array}$ & 65.3 & $\begin{array}{l}1.74, \mathrm{td} \\
(13.2,3.5)\end{array}$ & 30.2 \\
\hline $13 \mathrm{eq}$ & & & $1.27, \mathrm{~m}$ & \\
\hline 14 ax & $1.26, \mathrm{~m}$ & 31.3 & $0.85, \mathrm{~m}$ & 19.3 \\
\hline $14 \mathrm{eq}$ & $\begin{array}{l}1.96, \mathrm{td} \\
(13.0,3.4)\end{array}$ & & $\begin{array}{l}1.60, \text { br d } \\
(10.5)\end{array}$ & \\
\hline 15 & $\begin{array}{l}2.11, \text { br td } \\
(13.1,3.8)\end{array}$ & 44.4 & $1.87, \mathrm{~m}$ & 43.4 \\
\hline 16 & & 37.4 & & 37.3 \\
\hline 17 & $1.46, \mathrm{~s}$ & 24.3 & $1.43, \mathrm{~s}$ & 24.5 \\
\hline 18 & $1.04, \mathrm{~s}$ & 31.9 & $1.06, \mathrm{~s}$ & 31.5 \\
\hline 19 & $0.85, \mathrm{~s}$ & 19.6 & $0.74, \mathrm{~s}$ & 26.4 \\
\hline 20 & $\begin{array}{l}5.82, \mathrm{dd} \\
(17.1,11.0)\end{array}$ & 144.2 & $5.87, \mathrm{~m}$ & 147.7 \\
\hline 21 & $5.08, m$ & 114.0 & $4.87, \mathrm{~m}$ & 109.9 \\
\hline 22 & 8.57, br d (9.9) & $\mathrm{N}$ & $\begin{array}{l}8.23, \text { br d } \\
(9.16)\end{array}$ & N \\
\hline 23 & $8.04, d(1.37)$ & 159.7 & $\begin{array}{l}8.00, \text { br d } \\
(1.45)\end{array}$ & 159.6 \\
\hline
\end{tabular}

strengthen the key role of this functional group in regard to the bioactivity of members of the hapalindole family. Our work shows that antiproliferative effects on human T cells are more pronounced than toxicity on human $T$ cells, adding this activity to the wide range of reported bioactivities of the hapalindoles. The mode of action in human immune cells remains to be investigated. Moreover, concerning the fact that hapalindoles have been described to be neurotoxic metabolites [30], detailed studies which further discriminate toxicity and immunomodulatory effects need to be carried out in order to estimate whether or not a development as immunomodulatory drugs would be possible.
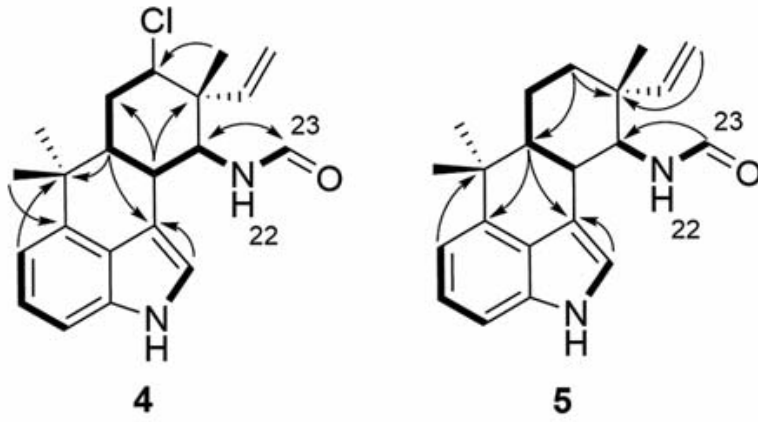

- Fig. $3{ }^{1} \mathrm{H}-{ }^{1} \mathrm{H}$ COSY (bold connections) and selected HMBC correlations (arrows) of 4 and 5.

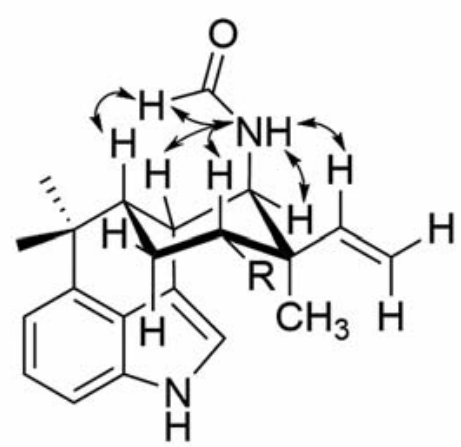

- Fig. 4 Selected NOESY correlations (arrows) of compounds 4 $(\mathrm{R}=\mathrm{Cl})$ and $5(\mathrm{R}=\mathrm{H})$.

\section{Materials and Methods}

\section{General experimental procedures}

HRESIMS data were obtained using a Q Exactive Plus mass spectrometer (Thermo Fisher Scientific) coupled to an UltiMate 3000 HPLC system (Thermo Fisher Scientific). Semi-preparative HPLC was conducted on an UltiMate $3000 \mathrm{HPLC}$ system (Thermo Fisher Scientific). NMR spectra were either recorded at $600 \mathrm{MHz}\left({ }^{1} \mathrm{H}\right.$ frequency) on a Bruker AV-III spectrometer using a cryogenically cooled $5 \mathrm{~mm}$ TCl-triple resonance probe equipped with 1-axis self-shielded gradients at $300 \mathrm{~K}$ or at $400 \mathrm{MHz}$ ( ${ }^{1} \mathrm{H}$ frequency) on an Agilent DD2 spectrometer. Spectra were referenced indirectly. If $1 \mathrm{D}$ spectra were not separately recorded, ${ }^{13} \mathrm{C}$ chemical shifts were extracted from the 2D spectra (compounds 1-3, and 5).

\section{Cyanobacterial material}

Hapalosiphon sp. CBT1235 was taxonomically identified as Hapalosiphon sp. on the basis of its morphology. The strain has kindly been provided by Algenol Biotech Inc. (USA) as ABCC 804 and is deposited in the culture collection of the Cyano Biotech $\mathrm{GmbH}$ 

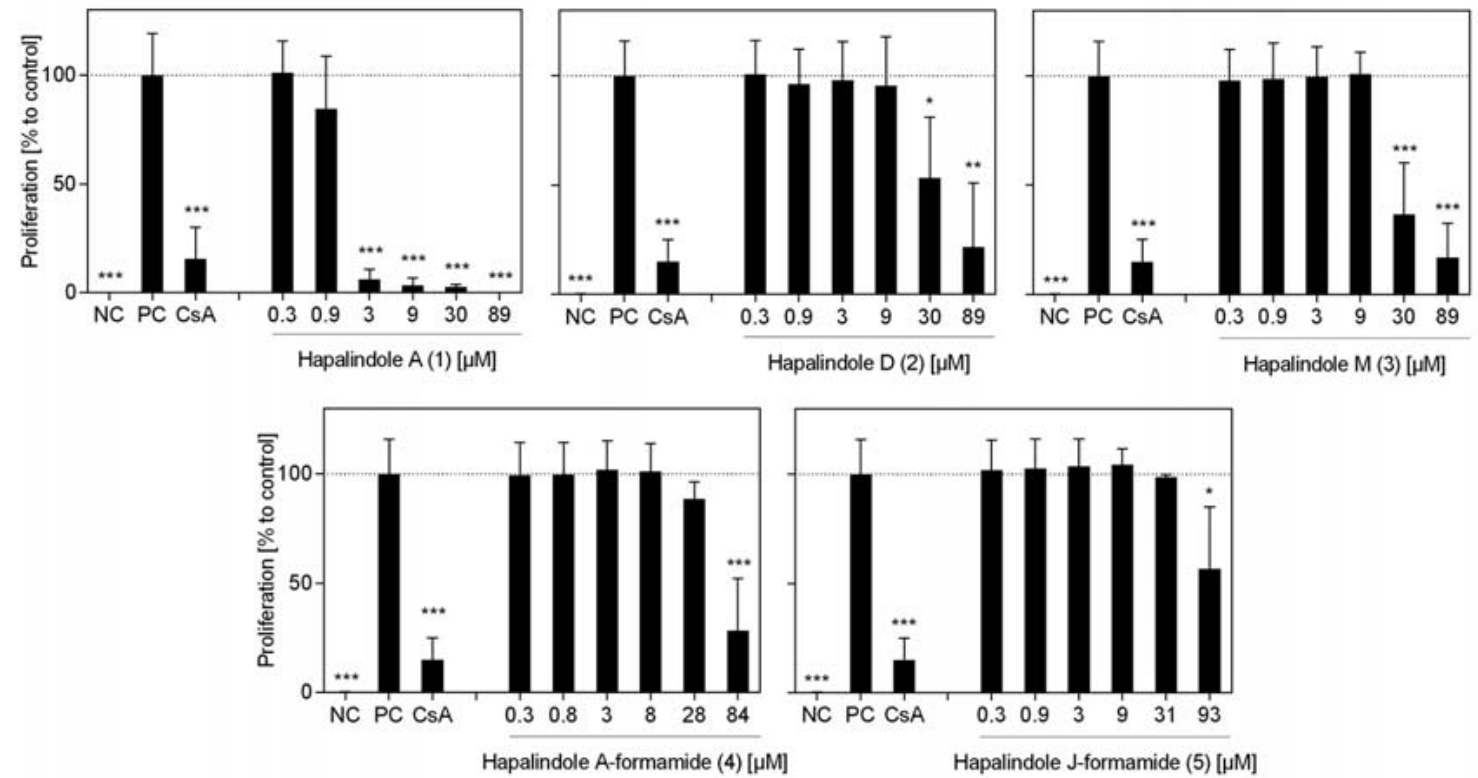

- Fig. 5 Effects of different hapalindoles on T cell proliferation. Primary human lymphocytes were cultured in the presence of medium (NC) or were stimulated with anti-human CD3 and anti-human CD28 mAb (PC; $100 \mathrm{ng} / \mathrm{mL})$. Activated T cells were further incubated with cyclosporine A (CsA; $5 \mu \mathrm{g} / \mathrm{mL}$ ) or different concentrations of 1-5. Cell division analysis was carried out using CFSE staining and flow cytometry. Data of 3 independent experiments are presented as mean $\pm S D$ in relation to stimulated T cells $(P C=100 \%)$. Asterisks indicate significant differences from PC controls $\left({ }^{* *} p<0.01,{ }^{* * *} p<0.001\right)$.

(Germany) under the accession number CBT 1235. The strain was cultivated in BG11 medium [39] at $28^{\circ} \mathrm{C}$ under continuous light $\left(60-80 \mu \mathrm{mol} \mathrm{m} \mathrm{m}^{2} \cdot \mathrm{s}^{-1}\right)$ in $20 \mathrm{~L}$ scale photobioreactors and harvested semi-continuously over a period of several weeks.

\section{Extraction, bioassay-guided fractionation, and isolation of compounds $1-5$}

Cyanobacterial cells were harvested and lyophilized. Seven grams dry biomass were suspended in $100 \mathrm{~mL} 50 \%$ methanol in water $(\mathrm{v} / \mathrm{v})$, treated with an ultrasonication rod (Bandelin), and extracted on a shaker for $30 \mathrm{~min}$ at room temperature. After centrifugation (20 min, $10800 \mathrm{~g}$ ), the biomass was extracted using $100 \mathrm{~mL} 80 \%$ methanol (v/v). The solutions were combined and dried under reduced pressure, yielding $0.4 \mathrm{~g}$ of biomass extract. For micro-fractionation, $4 \mathrm{mg}$ of extract were suspended in $0.1 \mathrm{~mL}$ acetonitrile and separated into 23 fractions by HPLC using a $C_{18}$ column $(250 \times 4.6 \mathrm{~mm}, 5 \mu \mathrm{m}, 100 \AA$, Luna, Phenomenex $)$ and $5-100 \%$ acetonitrile-water as the mobile phase at $1 \mathrm{~mL} / \mathrm{min}$ in $23 \mathrm{~min}$. All fractions were tested for inhibitory activity on T cell proliferation.

For preparative isolation of the active compounds, the remaining extract was dissolved in acetonitrile and fractionated by semipreparative HPLC using a phenyl-hexyl column $(250 \times 10 \mathrm{~mm}$, $5 \mu \mathrm{m}, 100 \AA$, Luna, Phenomenex) and 60-80\% acetonitrile-water as the mobile phase at $4.7 \mathrm{~mL} / \mathrm{min}$ in $25 \mathrm{~min}$ to afford $11 \mathrm{frac}$ tions. Fraction 1 ( $t_{R} 6.8 \mathrm{~min}$ ) was further purified by an additional round of semi-preparative HPLC using a phenyl-hexyl column (250× $100 \mathrm{~mm}, 5 \mu \mathrm{m}, 100 \AA$, Luna, Phenomenex) and 40-47\% acetonitrile-water as the mobile phase at $9.5 \mathrm{~mL} / \mathrm{min}$ in $20 \mathrm{~min}$ to afford hapalindole A-formamide $\left(4,5.0 \mathrm{mg}, \mathrm{t}_{\mathrm{R}} 16.5 \mathrm{~min}\right)$ and hapalindole J-formamide $\left(5,2.0 \mathrm{mg}, t_{R} 15.1 \mathrm{~min}\right)$. Fraction $6\left(t_{R}\right.$ $15.2 \mathrm{~min}$ ) was further purified by semi-preparative HPLC using a pentafluorophenyl column $(250 \times 100 \mathrm{~mm}, 5 \mu \mathrm{m}, 100 \AA$, Luna, Phenomenex) and 45-63\% acetonitrile-water as mobile phase at $9.5 \mathrm{~mL} / \mathrm{min}$ in $30 \mathrm{~min}$ yielding hapalindole $\mathrm{A}\left(1,16.1 \mathrm{mg}, t_{R}\right.$ $18.4 \mathrm{~min})$. No further purification was needed for fraction $8\left(t_{R}\right.$ $19.2 \mathrm{~min}$ ) and fraction $10\left(t_{R} 22.4 \mathrm{~min}\right)$, which corresponded to hapalindole $\mathrm{D}(2,5.3 \mathrm{mg})$ and hapalindole $\mathrm{M}(3,6.4 \mathrm{mg})$, respectively.

Hapalindole A (1): $[\alpha]_{\mathrm{D}}^{23}-64.2^{\circ}\left(\mathrm{CH}_{2} \mathrm{Cl}_{2}, \mathrm{c}\right.$ 1.2); HRESIMS (positive ion mode): $m / z 339.1616[\mathrm{M}+\mathrm{H}]^{+}$; NMR spectra, see Fig. 3S, 4S.

Hapalindole $D(2)$ : $[\alpha]_{\mathrm{D}}^{23}+45.2^{\circ}\left(\mathrm{CH}_{2} \mathrm{Cl}_{2}, \mathrm{c} 0.31\right)$; HRESIMS (positive ion mode): $\mathrm{m} / \mathrm{z} 337.1727[\mathrm{M}+\mathrm{H}]^{+}$; NMR spectra, see Fig. 5S, $6 \mathrm{~S}$.

Hapalindole $M(3)$ : $[\alpha]_{D}^{23}-6.7^{\circ}\left(\mathrm{CH}_{2} \mathrm{Cl}_{2}, \mathrm{c} 0.45\right)$; HRESIMS (positive ion mode): $m / z 337.1727[\mathrm{M}+\mathrm{H}]^{+}$; NMR spectra, see Fig. $7 \mathrm{~S}$, $8 \mathrm{~S}$.

Hapalindole A-formamide (4): brown oil; $[\alpha]_{\mathrm{D}}^{23}-56.2^{\circ}\left(\mathrm{CH}_{2} \mathrm{Cl}_{2}, \mathrm{C}\right.$ 0.45); UV (MeOH) $\lambda_{\max }(\log \varepsilon): 223$ (4.38), 282 (3.60), 292 (3.52)

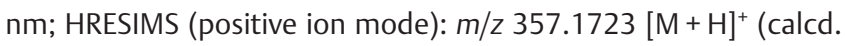
for $\mathrm{C}_{21} \mathrm{H}_{25} \mathrm{~N}_{2} \mathrm{OCl}$, 357.1728); NMR data, see $>$ Table 1; NMR spectra Fig. 9S-14S.

Hapalindole J-formamide (5): brown oil; $[\alpha]_{\mathrm{D}}^{23}+12.2^{\circ}\left(\mathrm{CHCl}_{3}, \mathrm{C}\right.$ 0.9); UV (MeOH) $\lambda_{\max }(\log \varepsilon): 224$ (4.24), 282 (3.50), 292 (3.41) nm; HRESIMS (positive ion mode): $\mathrm{m} / \mathrm{z} 323.2114[\mathrm{M}+\mathrm{H}]^{+}$(calcd. 

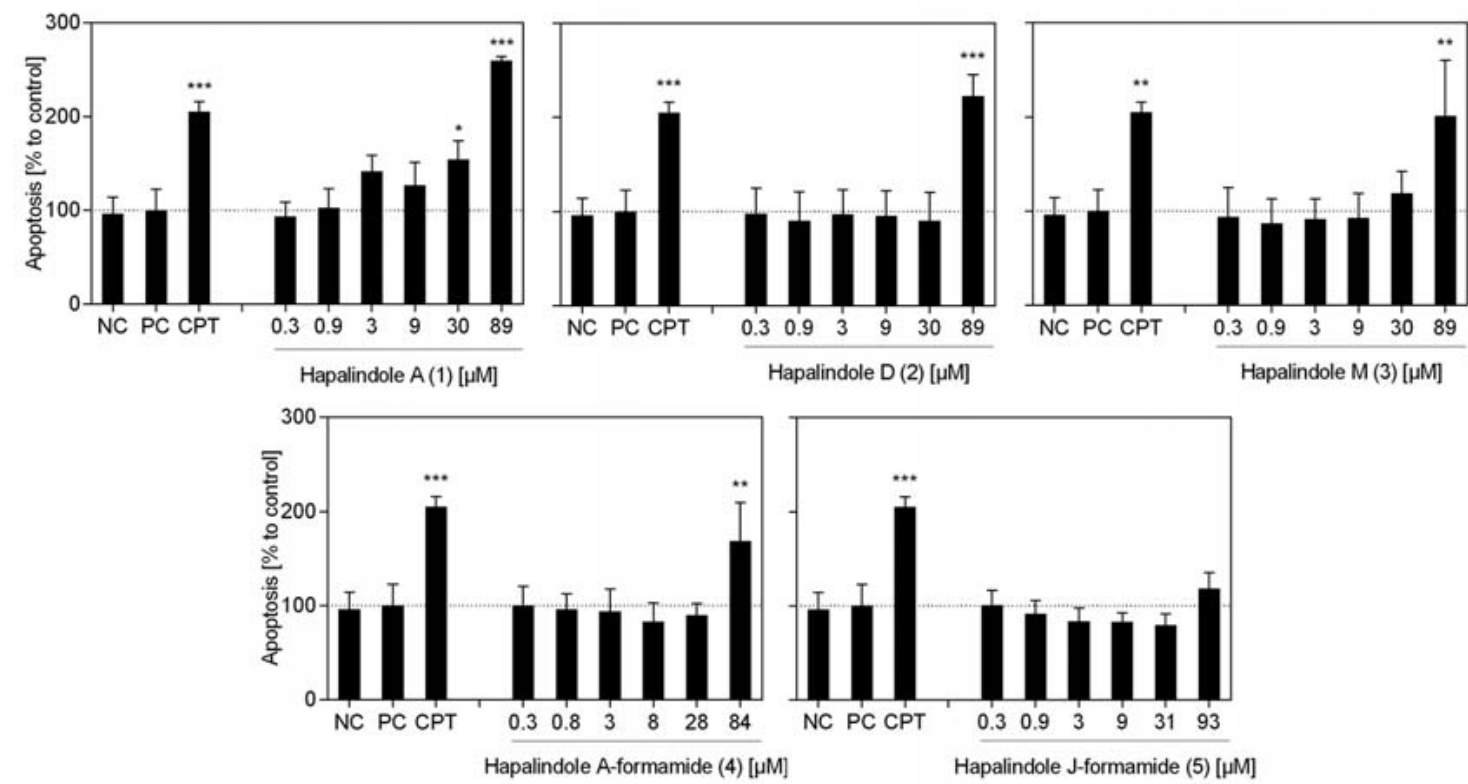

- Fig. 6 Levels of T cell apoptosis after treatment with different hapalindoles. Primary human lymphocytes were cultured in the presence of medium (NC) or were stimulated with anti-human CD3 and anti-human CD28 mAb (PC; $100 \mathrm{ng} / \mathrm{mL}$ ). Activated T cells were further incubated with camptothecin (CPT; $30 \mu \mathrm{g} / \mathrm{mL}$ ) or different concentrations of 1-5. Levels of apoptosis were determined using flow-cytometric analysis of annexin $V$-stained cells. Data of 3 independent experiments are presented as mean $\pm S D$ in relation to stimulated T cells $(P C=100 \%)$. Asterisks indicate significant differences from PC controls $\left({ }^{* *} p<0.01,{ }^{* * *} p<0.001\right)$.

for $\mathrm{C}_{21} \mathrm{H}_{26} \mathrm{~N}_{2} \mathrm{O}$, 323.2118); NMR data, see $>$ Table 1; NMR spectra Fig. 15S-19S.

\section{Ethics statement}

Written informed consent was obtained from patients prior to blood donation for research purposes. All experiments conducted on human material were approved by the ethics committee of the University Freiburg (55/14; February 11th, 2014).

\section{Preparation and cultivation of human immunocompetent cells}

Human PBMC were isolated from the blood of adult donors obtained from the Blood Transfusion Centre (University Medical Center Freiburg). Venous blood was centrifuged on a LymphoPrep gradient (density: $1.077 \mathrm{~g} / \mathrm{cm}^{3}, 20 \mathrm{~min}, 500 \mathrm{~g}, 20^{\circ} \mathrm{C}$; Progen). Afterwards, cells were washed twice with medium and cell viability and concentration was determined using the trypan blue exclusion test. Cells were cultured in Roswell Park Memorial Institute (RPMI) 1640 medium (Invitrogen) supplemented with 10\% heatinactivated fetal bovine serum (GE Healthcare), $2 \mathrm{mM}$ L-glutamine, $100 \mathrm{U} / \mathrm{mL}$ penicillin and $100 \mathrm{U} / \mathrm{mL}$ streptomycin (Invitrogen) at $37{ }^{\circ} \mathrm{C}$ in a humidified incubator with a $5 \% \mathrm{CO}_{2} / 95 \%$ air atmosphere. PBMC were additionally stimulated with anti-human CD3 (clone OKT3) and anti-human CD28 (clone 28.6) mAb (100 ng/mL; both from eBioscience). Incubation was carried out as indicated in the figure captions in the presence of medium alone, camptothecin (CPT; $30 \mu \mathrm{g} / \mathrm{mL}$; Tocris), cyclosporine A (CsA, $5 \mu \mathrm{g} / \mathrm{mL}$, Sandimmun $50 \mathrm{mg} / \mathrm{mL}$, Novartis), or different concentrations of the Hapalosiphon sp. CBT1235 extract (0.01, $0.1,1,10 \mu \mathrm{g} / \mathrm{mL}$ ), fractions (semi-quantitative using dilutions $1: 250,1: 500,1: 1000,1: 2000)$ or various hapalindoles $(0.1$, $0.3,1,3,10,30 \mu \mathrm{g} / \mathrm{mL})$.

\section{Cell division tracking using CFSE}

PBMC were harvested, washed twice in cold PBS (Invitrogen) and resuspended in PBS at a concentration of $5 \times 10^{6}$ cells $/ \mathrm{mL}$. CFSE ( $5 \mathrm{mM}$; Sigma) was diluted $1 / 1000$ and incubated for $10 \mathrm{~min}$ at $37^{\circ} \mathrm{C}$. The staining reaction was stopped by washing twice with complete medium. PBMC were activated as described above and cultured with the Hapalosiphon sp. CBT1235 extract, fractions, hapalindoles, or DMSO as a solvent control for $72 \mathrm{~h}$. Cell division progress was analyzed from 3 independent experiments with a BD FACSCalibur flow cytometer using BD CellQuest Pro Software.

\section{Determination of apoptosis and necrosis using annexin $\mathrm{V}$ and propidium iodide staining}

Cells were cultured as described, and levels of apoptosis and necrosis were determined using annexin V-FITC apoptosis detection kit (eBioscience) according to the manufacturer's instructions. After annexin $\mathrm{V}$ and propidium iodide staining, cells were analyzed by flow cytometry. CPT and Triton-X 100 (0.5\%; Sigma-Aldrich) were used as positive controls for apoptosis and necrosis, respectively. 


\section{Data analysis}

For statistical analysis, data were processed with Microsoft Excel and SPSS software (IBM, Version 22.0). Data were adjusted in relation to untreated control cells $(=100 \% \pm$ SD) and values are presented as mean \pm SD. Statistical significance was determined by one-way ANOVA followed by Dunnett's post hoc pairwise comparisons. $P$ values $<0.05$ were considered as statistically significant $\left({ }^{*} \mathrm{p}<0.05,{ }^{* *} \mathrm{p}<0.01,{ }^{* * *} \mathrm{p}<0.001\right) . \mathrm{IC}_{50}$ and $\mathrm{EC}_{50}$ values were determined using GraphPad Prism 6.

\section{Supporting information}

${ }^{1} \mathrm{H},{ }^{13} \mathrm{C}, \mathrm{HSQC}, \mathrm{HMBC}$ and NOESY spectra, MS data as well as a chromatogram of the micro-fractionation and the results of the inhibition of $\mathrm{T}$ cell proliferation by different Hapalosiphon sp. CBT1235 fractions are available as Supporting Information.

\section{Acknowledgements}

CG and CS have been financed by the Software AG foundation and DAMUS-DONATA e.V. Funding from the Federal Ministry of Education and Research (BMBF; project ANoBIn - 16GW0115) and the German Research Foundation (DFG; INST 271/388-1) for THJN is acknowledged. We thank Andrea Porzel and Peter Schmieder for recording the NMR spectra.

\section{Conflict of Interest}

$\mathrm{HE}$ is CSO and co-owner of Cyano Biotech GmbH, DE is CEO and co-owner of Cyano Biotech $\mathrm{GmbH}$. The company does not have any financial interest in the research presented here. The other authors declare no conflicts of interest. The funding sponsors had no role in the design, writing and publishing strategy of the study, nor in collection, analysis or interpretation of the data.

\section{References}

[1] Burja AM, Banaigs B, Abou-Mansour E, Burgess JG, Wright PC. Marine cyanobacteria - a prolific source of natural products. Tetrahedron 2001; 57: 9347-9377

[2] Dixit RB, Suseela MR. Cyanobacteria: potential candidates for drug discovery. Antonie Van Leeuwenhoek 2013; 103: 947-961

[3] Welker M, Dittmann E, von Döhren H. Cyanobacteria as a source of natural products. Methods Enzymol 2012; 517: 23-46

[4] Leao PN, Engene N, Antunes A, Gerwick WH, Vasconcelos V. The chemical ecology of cyanobacteria. Nat Prod Rep 2012; 29: 372-391

[5] Salvador-Reyes LA, Luesch H. Biological targets and mechanisms of action of natural products from marine cyanobacteria. Nat Prod Rep 2015; 32: 478-503

[6] Niedermeyer TH. Anti-infective natural products from cyanobacteria. Planta Med 2015; 81: 1309-1325

[7] Dittmann E, Gugger M, Sivonen K, Fewer DP. Natural product biosynthetic diversity and comparative genomics of the cyanobacteria. Trends Microbiol 2015; 23: 642-652

[8] Walton K, Berry JP. Indole alkaloids of the stigonematales (cyanophyta): chemical diversity, biosynthesis and biological activity. Mar Drugs 2016; 14: 73

[9] Moore RE, Cheuk C, Patterson GML. Hapalindoles: new alkaloids from the blue-green alga Hapalosiphon fontinalis. J Am Chem Soc 1984; 106: 6456-6457
[10] Moore RE, Cheuk C, Yang XQG, Patterson GML, Bonjouklian R, Smitka TA, Mynderse JS, Foster RS, Jones ND, Swartzendruber JK, Deeter JB. Hapalindoles, antibacterial and antimycotic alkaloids from the cyanophyte Hapalosiphon fontinalis. J Org Chem 1987; 291: 1036-1043

[11] Schwartz RE, Hirsch CF, Springer JP, Pettibone DJ, Zink DL. Unusual cyclopropane-containing hapalindolinones from a cultured cyanobacterium. J Org Chem 1987; 52: 3704-3706

[12] Moore RE, Yang XQG, Patterson GML, Bonjouklian R, Smitka TA. Hapalonamides and other oxidized hapalindoles from Hapalosiphon fontinalis. Phytochemistry 1989; 28: 1565-1567

[13] Park A, Moore RE, Patterson GML. Fischerindole L, a new isonitrile from the terrestrial blue-green alga Fischerella muscicola. Tetrahedron Lett 1992; 33: 3257-3260

[14] Smitka TA, Bonjouklian R, Doolin L, Jones ND, Deeter JB, Yoshida WY, Prinsep MR, Moore RE, Patterson GML. Ambiguine isonitriles, fungicidal hapalindole-type alkaloids from 3 genera of blue-green algae belonging to the Stigonemataceae. J Org Chem 1992; 57: 857-861

[15] Stratmann K, Moore RE, Bonjouklian R, Deeter JB, Patterson GML, Shaffer $\mathrm{S}$, Smith CD, Smitka TA. Welwitindolinones, unusual alkaloids from the blue-green algae Hapalosiphon welwitschii and Westiella intricata. Relationship to fischerindoles and hapalinodoles. J Am Chem Soc 1994; 116: 9935-9942

[16] Huber U, Moore RE, Patterson GML. Isolation of a nitrile-containing indole alkaloid from the terrestrial blue-green alga Hapalosiphon delicatulus. J Nat Prod 1998; 61: 1304-1306

[17] Asthana RK, Srivastava A, Singh AP, Singh SP, Nath G, Srivastava R, Srivastava BS. Identification of an antimicrobial entity from the cyanobacterium Fischerella sp. isolated from bark of Azadirachta indica (Neem) tree. J Appl Phycol 2006; 18: 33-39

[18] Becher PG, Keller S, Jung G, Süssmuth RD, Jüttner F. Insecticidal activity of 12-epi-hapalindole J isonitrile. Phytochemistry 2007; 68: 2493-2497

[19] Mo S, Krunic A, Chlipala G, Orjala J. Antimicrobial ambiguine isonitriles from the cyanobacterium Fischerella ambigua. J Nat Prod 2009; 72: 894-899

[20] Mo S, Krunic A, Santarsiero BD, Franzblau SG, Orjala J. Hapalindole-related alkaloids from the cultured cyanobacterium Fischerella ambigua. Phytochemistry 2010; 71: 2116-2123

[21] Kim H, Lantvit D, Hwang CH, Kroll D], Swanson SM, Franzblau SG, Orjala J. Indole alkaloids from 2 cultured cyanobacteria, Westiellopsis sp. and Fischerella muscicola. Bioorg Med Chem 2012; 20: 5290-5295

[22] Kim H, Krunic A, Lantvit D, Shen Q, Kroll D], Swanson SM, Orjala J. Nitrilecontaining fischerindoles from the cultured cyanobacterium Fischerella sp. Tetrahedron 2012; 68: 3205-3209

[23] Walton K, Gantar M, Gibbs PDL, Schmale MC, Berry JP. Indole alkaloids from Fischerella inhibit vertebrate development in the zebrafish (Danio rerio) embryo model. Toxins (Basel) 2014; 6: 3568-3581

[24] Raveh A, Carmeli S. Antimicrobial ambiguines from the cyanobacterium Fischerella sp. collected in Israel. J Nat Prod 2007; 70: 196-201

[25] Bhat V, Dave A, MacKay JA, Rawal VH. The chemistry of hapalindoles, fischerindoles, ambiguines, and welwitindolinones. Alkaloids Chem Biol 2014; 73: 65-160

[26] Klein D, Daloze D, Braekman JC, Hoffmann L, Demoulin V. New hapalindoles from the cyanophyte Hapalosiphon laingii. J Nat Prod 1995; 58: 1781-1785

[27] Doan NT, Rickards RW, Rothschild JM, Smith GD, Thanh Doan N. Allelopathic actions of the alkaloid 12-epi-hapalindole $\mathrm{E}$ isonitrile and calothrixin A from cyanobacteria of the genera Fischerella and Calothrix. J Appl Phycol 2000; 12: 409-416

[28] Doan NT, Stewart PR, Smith GD. Inhibition of bacterial RNA polymerase by the cyanobacterial metabolites 12-epi-hapalindole $\mathrm{E}$ isonitrile and calothrixin A. FEMS Microbiol Lett 2001; 196: 135-139 
[29] Becher PG, Jüttner F. Insecticidal compounds of the biofilm-forming cyanobacterium Fischerella sp. (ATCC 43239). Environ Toxicol 2005; 20: 363-372

[30] Cagide E, Becher PG, Louzao MC, Espiña B, Vieytes MR, Jüttner F, Botana LM. Hapalindoles from the cyanobacterium Fischerella: potential sodium channel modulators. Chem Res Toxicol 2014; 27: 1696-1706

[31] Bonjouklian R, Moore RE, Patterson GML. Acid-catalyzed reactions of hapalindoles. J Org Chem 1988; 53: 5866-5870

[32] Muratake H, Natsume M. Synthetic studies of marine alkaloids hapalindoles. Part I Total synthesis of ( \pm )-hapalindoles J and M. Tetrahedron 1990; 46: 6331-6342

[33] Schwartz E, Hirsch CF, Sesin DF, Flor JE, Chartrain M, Fromtling E, Harris $\mathrm{GH}$, Salvatore MJ, Liesch JM, Yudin K. Pharmaceuticals from cultured algae. J Ind Microbiol 1990; 5: 113-124
[34] Wagner MM, Shih C, Jordan A, Williams DC. In vitro pharmacology of cryptophycin 52 (LY355703) in human tumor cell lines. Cancer Chemother Pharmacol 1999; 43: 115-125

[35] Eggen M, Georg Gl. The cryptophycins: their synthesis and anticancer activity. Med Res Rev 2002; 22: 85-101

[36] Rohr J. Cryptophycin anticancer drugs revisited. ACS Chem Biol 2006; 1: 747-750

[37] Fukuyama T, Chen X. Stereocontrolled synthesis of (-)-hapalindole G. J Am Chem Soc 1994; 116: 3125-3126

[38] Chandra A, Johnston JN. Total synthesis of the chlorine-containing hapalindoles K, A, and G. Angew Chem Int Ed Engl 2011; 50: 7641-7644

[39] Andersen RA. Algal culturing Techniques. Burlington, MA: Elsevier Academic Press; 2005 